\title{
El bloqueo de la glucolisis colabora con la eficacia terapéutica de la inhibición de V600EBRAF en células tumorales tiroideas
}

\author{
Beatriz Gallego Tamayoa, Sergio Gago Díaz, Antonio Chiloeches Gálvez \\ a. bgallego28@gmail.com \\ IV Congreso de Señalización Celular, SECUAH 2019. \\ 20-22 de marzo, 2019. Universidad de Alcalá. Alcalá de Henares, Madrid. España. \\ Sesión A2, Cáncer.
}

Palabras clave: glucolisis; 2-Deoxyglucosa; V600EBRAF; cáncer; tiroides

\section{Resumen}

El cáncer de tiroides es el tumor endocrino más común y, aunque es altamente curativo, presenta una elevada tasa de recurrencia [1]. Una de las mutaciones más frecuentes en este tipo de tumores es la sustitución de la Valina 600 por un Glutámico (V600E) en la proteína BRAF. Esta mutación causa una hiperactivación de la vía de las ERK-MAPKs, favoreciendo la proliferación e invasividad tumoral e inhibiendo la muerte celular por apoptosis [2,3]. Sin embargo, las terapias con inhibidores de BRAF no dan los resultados esperados en el tratamiento del cáncer de tiroides, ya que, por una parte, estas células crean resistencia a este tratamiento y, por otra, causan importantes efectos adversos en la piel asociados a hiperproliferación llegando, en algunos casos, a producirse carcinomas de células escamosas y queratoacantomas en un $15-30 \%$ de los pacientes [4]. Debido a esto, se plantea la necesidad de utilizar terapias combinadas para aumentar la efectividad y especificidad del tratamiento en este tipo de cáncer. Por otra parte, las células tumorales reprograman su metabolismo energético por modificaciones en la expresión y actividad de enzimas metabólicas, para favorecer la proliferación acelerada. Por ello, el metabolismo de las células tumorales es una diana emergente para el tratamiento del cáncer, pudiendo usarse como terapia combinada con otros compuestos para aumentar la eficacia del tratamiento [5-8]. Estudios previos de nuestro laboratorio nos indican que la glucolisis es necesaria para la supervivencia de las células tumorales tiroideas con el oncogén V600EBRAF, por ello estudiamos la posibilidad terapéutica de combinar la inhibición glucolítica con la 2-Desoxyglucosa (2DG) y la inhibición de BRAF con PLX4720. Nosotros demostramos, en primer lugar, que tanto la inhibición del metabolismo de la glucosa, con el compuesto 2-Deoxyglucosa, como la inhibición del oncogén, disminuyen la captura de glucosa y producción de lactato, en la línea celular BHT101, derivada de un tumor anaplásico tiroideo. A continuación, estudiamos como la inhibición conjunta del oncogén V600EBRAF y del metabolismo glucolítico afectan a la viabilidad y supervivencia de las células BHT101 tanto in vitro, mediante distintas técnicas como MTT, capacidad de formación de colonias, TMRM, análisis del ciclo celular y determinación de células no viables positivas en ioduro de propidio por citometría de flujo; como in vivo, tras la generación de tumores xenografos en ratones nude tratados con los tratamientos individuales o en combinación. Los resultados obtenidos permiten concluir que la inhibición conjunta del oncogén V600EBRAF y la glucolisis aumenta la muerte celular in vitro, de forma independiente a apoptosis. Este efecto terapéutico también se observa in vivo en cuanto al tamaño tumoral en células anaplásicas tumorales tiroideas. Además, la modulación que ejercen sobre la glucolisis ambos tratamientos parece estar relacionada con cambios en la actividad de las proteínas implicadas en el metabolismos energético de las vías AMPK/ACC y mTOR/S6K, según muestran sus niveles de fosforilación medidos por WB. Finalmente, concluimos que la inhibición de la glucolisis con 2DG podría ser usado como terapia en los tumores tiroideos con la mutación V600EBRAF que no responden adecuadamente al tratamiento convencional con PLX4720.

Cita: Gallego Tamayo, Beatriz; Gago Díaz, Sergio; Chiloeches Gálvez, Antonio (2019) El bloqueo de la glucolisis colabora con la eficacia terapéutica de la inhibición de V600EBRAF en células tumorales tiroideas. Actas del IV Congreso de Señalización Celular, SECUAH 2019. 20-22 de marzo, 2019. Universidad de Alcalá. Alcalá de Henares, Madrid. España. Sesión A2, Cáncer. dianas 8 (1): e201903a21. ISSN 1886-8746 (electronic) journal.dianas.e201903a21 http://www3.uah.es/dianas?e201903a21.

URI http://hdl.handle.net/10017/15181

Copyright: @ Gallego-Tamayo B, Gago-Díaz S, Chiloeches-Gálvez A. Algunos derechos reservados. Este es un artículo open-access distribuido bajo los términos de una licencia de Creative Commons ReconocimientoNoComercial-SinObraDerivada 4.0 Internacional. http://creativecommons.org/licenses/by-nc-nd/4.0/ 